\section{The validity of the size-distance invariance hypothesis with cue reduction}

\author{
WALTER C. GOGEL \\ University of California, Santa Barbara, California 93106
}

The results from two recent studies, one by Epstein and Landauer (1969) and the other by Landauer and Epstein (1969) are interpreted by these authors as being in opposition to the size-distance invariance hypothesis and in agreement with the direct perception of retinal size. The present comments will provide an opposite interpretation of these data. It will be asserted that these studies support the size-distance invariance hypothesis and consequently are in opposition to the conclusion that a perception of size can occur independently of perceived distance.

The procedure in these studies was to obtain judgments of the size and distance of a disk of light, presented under reduced conditions of observation, either alone or simultaneously with a comparison disk. It is necessary, therefore, to examine the predictions made from the size-distance invariance hypothesis for these conditions. The size-distance invariance hypothesis states that a particular retinal size $(\theta)$ will determine a ratio of perceived size $\left(S^{\prime}\right)$ to perceived distance $\left(D^{\prime}\right)$. Or, most simply expressed,

$$
\mathrm{S}^{\prime} / \mathrm{D}^{\prime}=\mathrm{K} \theta
$$

where $\mathbf{K}$ is an observer constant. More generally, it is predicted that $S^{\prime} / D^{\prime}$ will be a monotonic increasing function of $\theta$. Predictions from these expressions of the size-distance invariance hypothesis will be applied to the study by Landauer and Epstein (1969). Also, if a variable (e) and standard disk (f) are presented simultaneously, as in the study by Epstein and Landauer (1969), it follows from Eq. 1 that

$$
\frac{\mathrm{S}_{\mathrm{e}}^{\prime} / \mathrm{S}_{\mathrm{f}}^{\prime}}{\mathrm{D}_{\mathrm{e}}^{\prime} / \mathrm{D}_{\mathrm{f}}^{\prime}}=\frac{\theta_{\mathrm{e}}}{\theta_{\mathrm{f}}}
$$

or, more generally, that the term on the left in Eq. 2 is a monotonic increasing function of $\theta_{\mathrm{e}} / \theta_{\mathrm{f}}$. Predictions from these expressions will be applied to the study by Epstein and Landauer (1969).

In the study by Epstein and Landauer, the standard disk subtended $1 \mathrm{deg} 12 \mathrm{~min}$ of visual angle at a constant distance. The variable disk also subtended $1 \mathrm{deg} 12 \mathrm{~min}$ in Condition 1 (with distance variable) or subtended different visual angels in
Conditions 2 and 3 (with distance variable in Condition 2 and constant in Condition 3). Since Conditions 2 and 3 provided different values of $\theta_{\mathrm{e}} / \theta_{\mathrm{f}}$, only the results from these conditions will be considered. The method of magnitude estimation was used, i.e., the size and distance of the standard were designated 10 and 0 assigned numbers to represent the size and distance of the variable judged relative to the standard. The only difference between Experiments 1 and 2 was that in the latter, unlike the former experiment, the size and distance reports were obtained as nearly simultaneously as possible. It was found from both experiments, under Conditions 2 and 3 , that reported distance decreased and reported size increased with increases in the visual angle of the variable disk. This result is interpreted by Epstein and

\section{Table 1}

Results from Transforming the Data of Table 1 by Epstein and Landauer (1969). $S_{e}^{\prime} a^{2}{ }_{d} S_{f}^{\prime}$ are the reported sizes, $D_{e}^{\prime}$ and $D_{f}^{\prime}$ are the reported distances, and $\theta_{e}$ and $\theta_{f}$ are the visual angles of the variable and standard disks, respectively.

Experiment 1, Condition 2

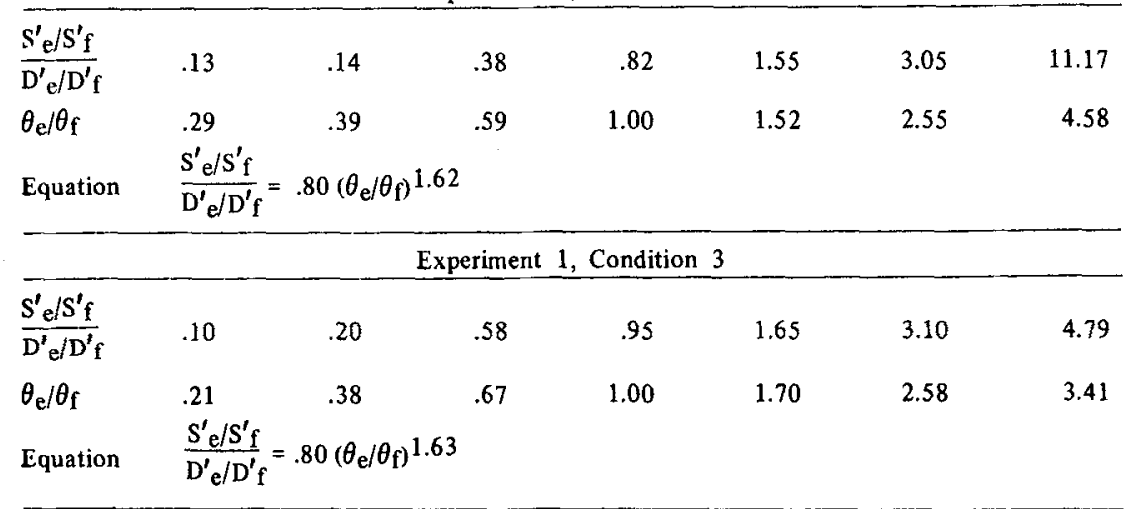

Experiment 2, Condition 2

\begin{tabular}{llllllll}
\hline $\mathrm{S}_{\mathrm{e}}^{\prime} / \mathrm{S}_{\mathrm{f}}^{\prime}$ & .20 & .38 & .54 & .96 & 1.40 & 2.56 & 6.68 \\
\hline $\mathrm{D}_{\mathrm{e}}^{\prime} / \mathrm{D}_{\mathrm{f}}^{\prime}$ & .20 & .59 & 1.00 & 1.52 & 2.55 & 4.58 \\
$\theta_{\mathrm{e}} / \theta_{\mathrm{f}}$ & .29 & .39 & .56 & & & \\
Equation & $\frac{\mathrm{S}_{\mathrm{e}}^{\prime} / \mathrm{S}_{\mathrm{f}}^{\prime}}{\mathrm{D}_{\mathrm{e}}^{\prime} / \mathrm{D}_{\mathrm{f}}^{\prime}}=.96\left(\theta_{\mathrm{e}} / \theta_{\mathrm{f}}\right)^{1.18}$ & & & & &
\end{tabular}

Experiment 2, Condition 3

\begin{tabular}{llllllll}
\hline $\mathrm{S}_{\mathrm{e}}^{\prime} / \mathrm{S}_{\mathrm{f}}^{\prime}$ & .14 & .27 & .58 & .95 & 1.64 & 2.71 & 3.86 \\
\hline $\mathrm{D}_{\mathrm{e}}^{\prime} / \mathrm{D}_{\mathrm{f}}^{\prime}$ & .14 & .37 & .67 & 1.00 & 1.70 & 2.58 & 3.41 \\
$\theta_{\mathrm{e}} / \theta_{\mathrm{f}}$ & .21 & .37 & & & & \\
Equation & $\frac{\mathrm{S}_{\mathrm{e}}^{\prime} / \mathrm{S}_{\mathrm{f}}^{\prime}}{\mathrm{D}_{\mathrm{e}}^{\prime} / \mathrm{D}_{\mathrm{f}}^{\prime}}=.90\left(\theta_{\mathrm{e}} / \theta_{\mathrm{f}}\right)^{1.18}$ & & & & \\
\hline
\end{tabular}


closely fitted by a linear function with the equations of the line of best fit shown in Table 1. According to the equations of Table 1, Eq. 2 should be expressed as the power function

$$
\frac{\mathrm{S}_{\mathrm{e}}^{\prime} / \mathrm{S}_{\mathrm{f}}^{\prime}}{\mathrm{D}_{\mathrm{e}}^{\prime} / \mathrm{D}_{\mathrm{f}}^{\prime}}=\left(\frac{\theta_{\mathrm{e}}}{\theta_{\mathrm{f}}}\right)^{\mathrm{n}}
$$

where $\mathbf{n}$ is greater than unity in Experiment 1 but close to unity in Experiment 2. The form of Eq. 1 that is consistent with Eq. 3 is

$$
S^{\prime} / D^{\prime}=K \theta^{n}
$$

The tendency for the coefficients in the equations of Table 1 to be different from unity very likely should be disregarded. If these differences were valid, it would indicate that $\mathrm{K}$ differed depending upon whether the judged disk was in the right or left portion of the visual field. In both experiments, the data are consistent with the general form of the size-distance invariance hypothesis. It can be concluded that the simultaneous increase in reported size and decrease in reported distance with increasing retinal size occurring in the study by Epstein and Landauer is consistent with, rather than in opposition to, the size-distance invariance hypothesis.

In the study by Landauer and Epstein (1969), one disk at a time was presented with disks of different angular sizes presented to different groups of Os. Again it was found that reported distance decreased while reported size increased with increases in visual angle, and again the authors conclude that the data are in opposition to the size-distance invariance hypothesis. The authors note, however, that "the mean visual angle equivalents were $0.351,1.24,3.94$, and $6.89 \mathrm{deg}$ for the 1-, 2-, 4-, and 8-deg standard, respectively [p. 274]." Since the mean visual angle equivalents are mean values of $S^{\prime} / D^{\prime}$, it follows that for each increase in $\theta$ an increase occurred in $S^{\prime} / D^{\prime}$. A log-log plot of these data are reasonably fitted by a linear function with the equation of the line of best fit given by $S^{\prime} / D^{\prime}=.41 \theta^{1.46}$. These results support the general form of the size-distance invariance hypothesis (Eq. 4) with $n$ greater than unity. The data from both of these studies, instead of being opposed to the size-distance invariance hypothesis, provide strong support for this hypothesis, at least in its general form. Thus, these studies do not provide support for the conclusion that a retinal size can determine a perceived size independent of a perceived distance. As suggested by Coltheart (1968, p.41), a simultaneous decrease in perceived size and increase in perceived distance is not necessarily in opposition to the size-distance invariance hypothesis.

Also, caution is necessary before it can be concluded from these studies that the exponent $\mathbf{n}$ in Eqs. 3 and 4 is greater than unity. One difficulty is that the instructions to $O$ seemed to suggest that $O s$ were to judge physical rather than perceived size and distance, whereas $S^{\prime}$ and $D^{\prime}$ in the above equations represent perceived extents. Also, in the study by Epstein and Landauer the values of $\mathbf{n}$ were close to unity in Experiment 2. But Experiment 2, rather than Experiment 1, probably represents a better test of the size-distance invariance hypothesis since, in Experiment 2, the size and distance judgments were obtained nearly simultaneously. Finally, if Eq. 4, rather than Eq. 1, were correct, it would follow that the perceived sizes of two objects at the same apparent distance $\left(D_{e}^{\prime} \doteq D_{f}^{\prime}\right)$ would not be a linear function of retinal size. Although it has been found (Teghtsoonian, 1965) that this result can occur if $\mathrm{O}$ is responding to area rather than to linear extent, the exponent of the power function under these conditions is less than, not greater than, unity. On the other hand, values of $n$ greater than unity have been obtained by Foley (1968) using reduced binocular conditions and size-distance ratio judgments.

The size-distance invariance hypothesis in its general form predicts the interdependence of perceived size and perceived distance obtained in these studies without specifying the conditions under which changes in one of these terms (with concomittant changes in the other) will occur. There is a need to explain, thercfore, why the reported size of the variable relative to the standard disk changed in Conditions 2 and 3 in the study by Epstein and Landauer. Normally, from the relative size cue it would be expected that changes in retinal size between the two objects would result in changes in perceived distance with the perceived sizes remaining constant. A possible explanation occurs in terms of the equidistance tendency (Gogel, 1965). It is expected from the equidistance tendency that under relatively reduced conditions of observation (as occurs when the relative size is the only distance cue available) the perceived relative depth between the two disks would be underestimated. As a consequence, and in agreement with the size-distance invariance hypothesis, both the ratio of perceived sizes and the ratio of perceived distances would differ from the ratio of retinal sizes.

The same kind of explanation cannot be applied, however, to the study by
Landauer and Epstein, since in this case only one object was present at a time and the different retinal sizes were presented to different Os. How are the simultaneous changes in reported size and distance as a function of retinal size to be explained in this study? The authors of this study suggest that the retinal size was a separate determiner of each of these judgments. Although the results are consistent with the size-distance invariance hypothesis, this consistency does not prove that the interpretation by Landauer and Epstein is invalid. Although unlikely, it is possible that the retinal size independently determined perceived size and perceived distance, with each of these independent functions being such as to produce results that satisfied the size-distance invariance hypothesis.

Landauer and Epstein have pointed out that in the study by Gogel (1969), in which different sizes of rectangles were presented to different groups of Os (with "apparent" instructions), perceived size, but not perceived distance, varied significantly with retinal size. Although not significant, the changes in perceived distance were, however, in the direction of those obtained by Landauer and Epstein. Epstein (1967), in a condition preliminary to a study of perceptual learning, also obtained significant changes in judged size but not in judged distance. On the other hand, Coltheart (1968), using objective instructions, found judged distance but not judged size to vary significantly with retinal size. In a recent study by Gogel and Sturm," using "apparent" instructions, significant changes in perceived size and sometimes in perceived distance were obtained. Some of the data from this last study, unlike those obtained in the above experiments, were not in agreement with the size-distance invariance hypothesis.

It is suggested that these differing results can be explained in terms of the following process. $^{2}$ There is evidence that, under reduced conditions of observation, objects will tend to be perceived at a relatively near distance (Gogel, 1969). This has been termed the specific distance tendency. Superimposed upon the operation of this tendency are such residual cues as accommodation and accommodative convergence, which to some extent probably are unavoidably present in all experiments. The combination of the specific distance tendency and residual cues provide a perceived distance and, in agreement with the size-distance invariance hypothesis, the perception of size at this constant perceived distance is proportional to retinal size. This perceived size, termed $\mathrm{S}_{\theta}^{\prime}$, al though proportional to retinal size, is the result of perceiving the object at the 
distance determined by the specific distance tendency (a perceived distance, $\left.\mathrm{D}_{\mathrm{T}}^{\prime}\right)$ and is consistent with the relation between perceived size and perceived distance expressed by the size-distance invariance hypothesis.

If the object presented under reduced conditions is a familiar object, it will, in addition to $S_{\theta}^{\prime}$, have a familiar size, $S_{F}^{\prime}$. It has been demonstrated (Gogel, 1969; Gogel \& Newton, 1969) that if $S_{\theta}^{\prime}$ and $S_{F}^{\prime}$ are sufficiently different the familier object will be perceived as off-sized. Depending upon the direction of the difference between $S_{\theta}^{\prime}$ and $S_{F}^{\prime}$, the familiar object will be seen as a small or a large off-sized object. But, it has also been demonstrated that $O$ expects a distant object to appear smaller than a near object of equal physical size (Carlson, 1960; Epstein, 1963). It follows that $\mathrm{O}$ will infer that an object that appears smaller than normal is distant and that an object that appears larger than normal is nearby. Thus, when asked for a judgment of the distance of perceptually off-sized objects, $O$ will tend to report, for example, that the apparently small off-sized object is far away. It has been shown (Gogel, 1969) that the magnitude of this inferred judgment $\left(D_{1}\right)$ is inversely related to the magnitude of the off-sized appearance, i.e., to the magnitude of $S_{\theta}^{\prime} / S_{F}^{\prime}$. It is suggested that the same process occurs for so-called nonrepresentative objects, such as a disk or rectangle. When presented with such objects $O$ is not entirely neutral with respect to an assumed (familiar) size, i.e., an $S_{F}^{\prime}$ as well as an $S_{\theta}^{\prime}$ probably occurs. The value of $S_{F}^{\prime}$ for nonrepresentative objects, however, would be expected to be less precisely determined than in the case of more representative objects, and an inferrential distance judgment would be less likely to occur.
When presented with either a representative or a nonrepresentative object, $O$ has two sources of size information available, i.e., $S_{\theta}^{\prime}$ and $S_{F}^{\prime}$. There are also two sources of distance information, i.e., a perceived distance, $\mathrm{D}^{\prime} \mathrm{T}$, from the specific distance tendency and an inferred distance, $D_{\mathbf{I}}$, from the off-sized perceptions. Various combinations of these sources can be used in determining the reports. The following combinations would produce results that are consistent with the size-distance invariance hypothesis: (1) $\mathrm{S}_{\theta}^{\prime}$ and $D_{T}^{\prime},(2) S_{F}^{\prime}$ and $D_{I}$, (3) a weighted average of $S_{\theta}^{\prime}$ and $S_{F}^{\prime}$ and the same weighted average of $D^{\prime} T$ and $D_{I}$. It will be noted that Combination 3 would produce the results obtained by Landauer and Epstein, Combination 1 would tend to produce the results obtained by Gogel (1969), and Combination 2 would produce the results obtained by Coltheart (1968). On the other hand, the strategy of using $S_{\theta}^{\prime}$ together with $D_{I}$ or of using $S_{F}^{\prime}$ together with $\mathrm{D}_{T}^{\prime}$ would produce results seemingly inconsistent with the size-distance invariance hypothesis even in its general form. According to this explanation a number of judgmental alternatives are available to 0 , even though from the viewpoint of $E$ the stimulus conditions are relatively or totally reduced. It must be emphasized, however, that these alternatives are available because of the occurrence of the basic perceptual process indicated by $S_{\theta}^{\prime}$ and $D_{T}^{\prime}$ with this process consistent with the size-distance invariance hypothesis. Thus, the size-distance invariance hypothesis provides a description of a perceptual process upon which experiential and inferfential factors can be readily superimposed to provide a flexible system of responding to size and distance information with the response strategy possibly affected by the set of $O$.

\section{REFERENCES}

CARLSON, V. R. Overestimation in size-constancy judgments. American Joumal of Psychology, 1960, 73, 199-213.

COLTHEART, M. Size information as a factor in visual judgments of absolute distance under reduced conditions. Doctoral dissertation, University of Sydney, Australia, 1968.

EPSTEIN, W. Attitudes of judgment and the size-distance invariance hypothesis. Joumal of Experimental Psychology, 1963, 66, 78-83.

EPSTEIN, W. Perceptual learning resulting from exposure to a stimulus invariant. American Journal of Psychology, 1967, 80, 205-212.

EPSTEIN, W., \& LANDAUER, A. A. Size and distance judgments under reduced conditions of viewing. Perception \& Psychophysics, 1969, 6, 269-272.

FOLEY, J. M. Depth size and distance in stereoscopic vision. Perception \& Psychophysics, 1968, 3, 265-274.

GOGEL, w. C. Equidistance tendency and its consequences. Psychological Bulletin, 1965, 64, 153-163.

GOGEL, W. C. The effect of object familiarity on the perception of size and distance. Quarteriy Journal of Experimental Psychology, 1969 , 21, 239-247.

GOGEL, W. C., \& NEWTON, R. E. Perception of off-sized objects. Perception \& Psychophysics, $1969,5,7-9$.

LANDAUER, A. A., \& EPSTEIN, W. Does retinal size have a unique correlate in perceived size. Perception \& Psychophysics, 1969, 6, 273-275.

TEGHTSOONIAN, M. The judgment of size. Amcrican Journal of Psychology, 1965, 78, 392-402.

\section{NOTES}

1. Gogel, W. C., \& Sturm, R. Directional separation and the size cue to distance. In preparation.

2. Gogel, W. C. The organization of perceived space. In preparation.

(Accepted for publication May 15, 1970.) 